\title{
Modelling the Austenite to Ferrite Phase Transformation in Low Carbon Steels in Terms of the Interface Mobility
}

\author{
T. A. KOP, Y. van LEEUWEN, Jilt SIETSMA and S. van der ZWAAG \\ Laboratory for Materials Science, Rotterdamseweg 137, 2628 AL Delft, The Netherlands. E-maili: J.Sietsma@TNW.TUDELFT.NL
}

(Received on December 13,1999; accepted in final form on February 18, 2000)

\begin{abstract}
The kinetics of the phase transformation between the high temperature FCC-phase austenite and the low temperature BCC-phase ferrite as it occurs during controlled cooling of hot rolled low carbon steels is described using a physical model that considers austenite grain size, nucleus density, composition effects, and the austenite/ferrite interface mobility. The model is verified against experimental dilatometry data for three lean carbon-manganese steel grades. The model yields adequate reproductions of the transformation kinetics. Ferrite grain coarsening during the transformation appears to have a significant effect on the final microstructure.
\end{abstract}

KEY WORDS: $\quad$ transformation model; transformation kinetics; interface mobility; low-carbon steel.

\section{Introduction}

A crucial step in the production process of low-carbon steel strip is the decomposition of austenite during cooling after hot rolling. It is during this phase transformation that the microstructure is formed. The microstructure determines the properties of the material, not only by means of the phases present, but also through the dimensions (grainsize distribution, morphology) of the microstructural components. Being a thermally activated process of nucleation and growth, the phase transformation strongly depends on the time/temperature profile to which the material is subjected. In general, the occurrence of an undercooling due to fast cooling results in an enhanced nucleation rate at lower temperatures, which in turn leads to a fine ferritic/pearlitic microstructure, of which the grain sizes are also influenced by the grain-coarsening process. The formation of these phases, depending on a balance between nucleation rate and growth rate, is a complex process, difficult to control. In order to improve the control of the microstructure formation in low-carbon steels, several types of models have been developed to describe the kinetics of the austenite decomposition.

A number of empirical or semi-empirical models has been presented in the literature, of which the description of transformation kinetics by the Johnson-Mehl-Avrami scheme $^{1,2)}$ is among the most widely applied. A model that is more physically based, relating to the kinetics of carbon diffusion during the transformation, is the almost classical model developed by Zener. ${ }^{3)}$ This model takes into account that, in the case of alloys with a carbon content above 0.02 $\mathrm{wt} \%$, the ferrite phase cannot contain all the carbon. Consequently, at the ferrite/austenite interface the austenite enriches in carbon. Zener assumes this pile-up of carbon to result in local equilibrium at the ferrite/austenite interface and models the phase-transformation kinetics by assuming the carbon diffusion in the austenite phase to be rate determining. An implicit assumption in this model is that the actual lattice transformation from FCC austenite to BCC ferrite is fast enough to be of no significance to the transformation rate. Notwithstanding the great merits of this diffusion-control model, it is not suited to describe the phase transformation in ultra-low carbon steels and interstitialfree steels, which are becoming increasingly important. Evidently in these steels carbon diffusion plays a less prominent role, so for adequate modelling it is necessary to take the kinetics of the lattice transformation itself into account. The concept of interface mobility, reflecting these kinetics, has been defined in general terms by Christian. ${ }^{4)}$

The interface mobility determines the interface velocity as a function of the driving force for transformation given by the Gibbs free-energy difference between the austenite and ferrite phases. In most commonly used alloys the transformation rate is determined by the balance between interface mobility and carbon diffusion, leading to so-called mixed-mode models accounting for both these phenomena. An example of a phase-transformation model incorporating the influence of the interface mobility on the kinetics of the austenite-to-ferrite transformation can be found in recent work by Krielaart et al. ${ }^{5)}$ In this model, the interface velocity is assumed to be proportional to the local driving force for transformation, which in turn is dependent on both the development of the transformation and the diffusion characteristics. In order to "translate" the interface velocity to macroscopic transformation kinetics, the initial austenite microstructure is modelled by a model austenite grain hav- 
ing the shape of a tetrakaidecahedron, and ferrite is assumed to nucleate at grain corners and grain boundaries. ${ }^{6}$ ) The model has been shown to yield good results for the phase transformation in iron alloys containing substitional alloying elements. ${ }^{7}$

In the present paper the phase-transformation kinetics at different cooling rates are determined for three commercial low-alloy steels with carbon contents between 0.05 and $0.10 \mathrm{wt} \%$. The measurements are performed by means of dilatometry, and the resulting development of the fraction ferrite as a function of temperature during cooling is simulated by means of the interface mobility model. An assessment of the influence of carbon diffusion, transformation stresses, and ferrite nucleation and grain coarsening is made.

\section{Theory}

The factors playing a role in the austenite $(\gamma)$ to ferrite $(\alpha)$ phase transformation can be divided into three categories: (1) nucleation and coarsening, determining the ferrite grain density; (2) interface migration, and (3) geometry of the austenite and the ferrite. This section will discuss the model in terms of these three features.

\subsection{Nucleation}

Nucleation is a process that is experimentally difficult to investigate. Extensive studies have been performed among others by Enomoto and Aaronson, ${ }^{8)}$ regarding the so-called classical nucleation theory. Notwithstanding these scientific efforts, many aspects concerning the nucleation kinetics still lack experimental evidence. An additional complication for studies on nucleation is caused by grain coarsening. During the last decade several papers have been published concerning the question of the coarsening of ferrite grains during the transformation. Priestner and Hodgson ${ }^{9)}$ investigated the coarsening behaviour of a deformed niobium steel and indicated that the coarsening of ferrite grains already starts in the beginning of the transformation. Bengochea et $a l{ }^{10)}$ show that at least in the later stages of the transformation the competition between coarsening and the kinetics of the formation of ferrite are important in determining the final grain size. This conclusion was also based on niobium containing steels with retained strain being present at the start of the transformation. From their work it follows that if the nucleus density is estimated from the final microstructure an error of at least one order of magnitude is made. Hodgson et al. ${ }^{11)}$ indicate that in the absence of niobium (and at low retained strains) the nucleation - instead of coarsening - may be the decisive step in determining the final ferrite grain size. This conclusion was obtained by comparing ferritic grain sizes from continuous and discontinuous cooling experiments on low carbon steels.

In the present paper, the nucleus density is derived from optical microscopy on both the austenite and the final ferrite microstructure. From the average grain sizes in both microstructures, the number ratio of austenite and ferrite grains, and hence the number of nuclei per austenite grain, is deduced. In applying this scheme, we assume as a first approximation that grain coarsening does not occur in the ferrite. It is assumed that nucleation is taking place instan- taneously at a certain temperature, viz. upon entering the two-phase region, where the austenite becomes instable; in other words, no undercooling due to nucleation is introduced.

In the model austenite grain, the ferrite nuclei are positioned at grain corners, assuming heterogeneous nucleation to be predominant (see Sec. 2.3).

\subsection{Interface Migration}

A detailed description of the progression of the interface during the transformation requires a profound knowledge of the local conditions at the interface. However, since these conditions cannot be monitored in detail as a function of time and temperature, for the model the choice has been made to link the transformation kinetics to the average thermodynamical properties of the bulk of the material. The model used to accomplish this was first introduced by Christian. ${ }^{4)}$ Christian derives the net frequency $\omega$ of atoms jumping from the austenite region to the ferrite region to be given by

$$
\omega=f^{*} \exp \left(-\frac{Q}{R T}\right)\left[1-\exp \left(\frac{\Delta G^{\gamma / \alpha}}{R T}\right)\right],
$$

where $f^{*}$ is the characteristic frequency of the atoms, $R$ the gas constant, $T$ the temperature, $Q$ the activation energy and $\Delta G^{\gamma / \alpha}$ the Gibbs free-energy difference between the austenite and ferrite phases, taking into account their compositions. Since every atom jumping across the interface shifts the interface locally over a distance $\delta$, the atomic diameter, the interface velocity $v$ can be calculated according to

$$
\nu=\omega \cdot \delta=M_{0} \exp \left(-\frac{Q}{R T}\right) \cdot \Delta G^{\gamma / \alpha},
$$

using the condition that $\Delta G^{\gamma / \alpha} \ll R T . M_{0}=f^{*} \delta / R T$ is a preexponential factor describing the mobility of the interface.

All in all, the model uses three quantities to describe the phase transformation: the activation energy $Q$, the free-energy difference $\Delta G^{\gamma / \alpha}$ and the pre-exponential factor $M_{0}$. The activation energy $Q$ is expected to be similar to the activation energy for grain-boundary diffusion, as it is the energy barrier for atoms "entering" the interface. Its value is expected to be comparable to the value for recrystallisation in pure iron. Hillert experimentally determined this value to be $147 \mathrm{~kJ} / \mathrm{mol}^{12)}$ In a study by Krielaart and Van der Zwaag $^{13)}$ into the transformation behaviour of binary $\mathrm{Fe}$ Mn alloys a similar value $(140 \mathrm{~kJ} / \mathrm{mol})$ was found.

For the calculation of the driving force $\Delta G^{\gamma / \alpha}$, for which the thermodynamic database programme MTData is used, the alloys are treated as $\mathrm{Fe}-\mathrm{C}-\mathrm{Mn}$ systems. Since the diffusion rate of manganese atoms is much lower than that of carbon atoms, it is assumed that only the carbon in the system redistributes during the transformation, while the manganese content remains equal in both the ferrite and the austenite phase. This situation is often referred to as paraequilibrium. It implies that the $\mathrm{Fe}-\mathrm{Mn}$ atom ratio in the two phases remains constant throughout the transformation, which reduces the system to a quasi-binary system $\mathrm{M}-\mathrm{C}$, where $\mathrm{M}$ stands for a metal atom, either Fe or Mn.

In order to calculate the driving force for the transforma- 


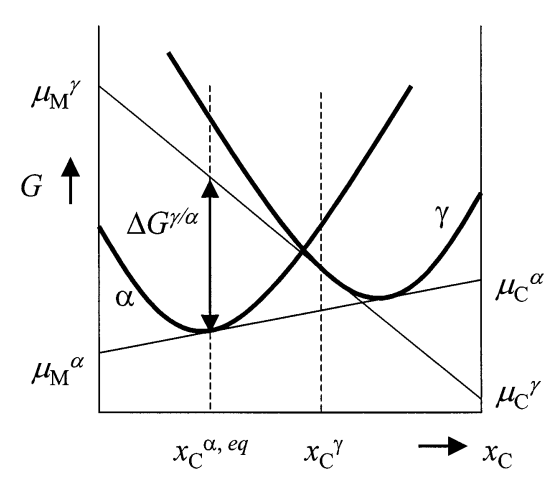

Fig. 1. Schematic representation of the determination of the driving force for transformation, $\Delta G^{\gamma / \alpha}$.

tion, the chemical potentials of the atoms are considered. The summation of the chemical potentials of all the elements constituting a system yields the free energy of the system. Under the assumption of para-equilibrium this summation can be written as

$$
G^{i}=\left(1-x_{\mathrm{C}}^{i}\right) \mu_{\mathrm{M}}^{i}\left(x_{\mathrm{C}}^{i}\right)+x_{\mathrm{C}}^{i} \mu_{\mathrm{C}}^{i}\left(x_{\mathrm{C}}^{i}\right),
$$

with $i$ being either $\alpha$ or $\gamma$, and $\mu_{\mathrm{M}}^{i}$ and $\mu_{\mathrm{C}}^{i}$ the chemical potentials of the metal and the carbon atoms respectively. $\mu_{\mathrm{M}}^{i}$ and $\mu_{\mathrm{C}}^{i}$ for a certain phase and composition can be obtained by taking the tangent of the free-energy curve of the phase in question at the appropriate composition (Fig. 1). The values of this tangent at $x_{\mathrm{C}}=0$ and $x_{\mathrm{M}}=0$ provide $\mu_{\mathrm{M}}$ and $\mu_{\mathrm{C}}$ respectively. The driving force for transformation can be calculated by summing the differences in the chemical potentials in $\alpha$ and $\gamma$ for the atoms crossing the interface, according to

$$
\Delta G^{\gamma / \alpha}=x_{\mathrm{C}}^{\gamma / \alpha} \Delta \mu_{\mathrm{C}}+\left(1-x_{\mathrm{C}}^{\gamma / \alpha}\right) \Delta \mu_{\mathrm{M}}
$$

where $x_{\mathrm{C}}^{\gamma / \alpha}$ is the carbon fraction of the atoms crossing the $\gamma / \alpha$-interface, and $\Delta \mu_{\mathrm{A}}=\mu_{\mathrm{A}}^{\gamma}\left(x_{\mathrm{C}}^{\gamma}\right)-\mu_{\mathrm{A}}^{\alpha}\left(x_{\mathrm{C}}^{\alpha}\right)$. The composition of the atom flux crossing the $\gamma / \alpha$-interface is equal to the composition in the ferrite phase, which is assumed to be equal to the equilibrium concentration $x_{\mathrm{C}}^{\alpha, \text { eq }}$. The carbon content in the austenite phase $x_{\mathrm{C}}^{\gamma}$ is assumed to be homogeneous throughout the whole phase and is calculated by

$$
x_{\mathrm{C}}^{\gamma}=\frac{x_{0}-f^{\alpha} x_{\mathrm{C}}^{\alpha, \text { eq. }}}{1-f^{\alpha}},
$$

with $x_{0}$ the average carbon concentration of the alloy and $f^{\alpha}$ the ferrite fraction. This method is commonly referred to as the "mean field" approach. It should be noted that $x_{C}^{\gamma}$ changes substantially during the transformation, while $x_{\mathrm{C}}^{\alpha, \text { eq }}$ remains roughly constant at $0.02 \mathrm{wt} \%$. In this paper the preexponential factor $M_{0}$ is obtained by fitting the model to experimentally determined macroscopic data.

\subsection{Geometry Model}

Equation (2) describes the velocity of an $\alpha / \gamma$-interface. In order to verify this equation, experimental data on the phase transformation are needed, for which dilatometry is used in this study. This method yields the volume fraction ferrite formed from austenite as a function of temperature. In order to calculate the three-dimensional volume fraction

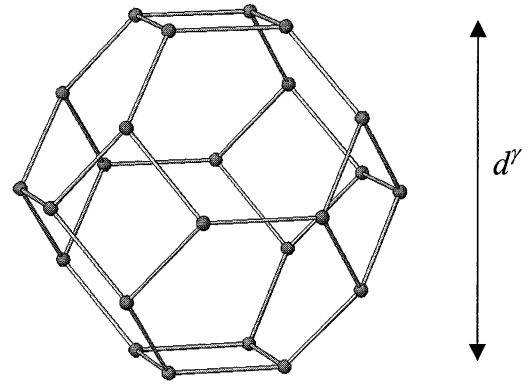

Fig. 2. A tetrakaidecahedron as the model representation of an austenite grain.

Table 1. Compostitions of the alloys used in this investigation in $\mathrm{wt} \%(\mathrm{at} \%)$.

\begin{tabular}{|l|c|c|c|c|c|c|c|c|c|c|c|}
\hline & $\mathrm{C}$ & $\mathrm{Mn}$ & $\mathrm{Si}$ & $\mathrm{Cu}$ & $\mathrm{Cr}$ & $\mathrm{Ni}$ & $\mathrm{Mo}$ & $\mathrm{Sn}$ & $\mathrm{P}$ & $\mathrm{S}$ & $\mathrm{N}$ \\
\hline alloy A & 0.103 & 0.490 & 0.006 & 0.009 & 0.018 & 0.021 & 0 & 0 & 0.010 & 0.014 & 0 \\
& $(0.48)$ & & & & & & & & & & \\
\hline alloy B & 0.072 & 0.365 & 0.007 & 0.010 & 0.024 & 0.024 & 0.002 & 0 & 0.012 & 0.013 & 0 \\
& $(0.33)$ & & & & & & & & & & \\
\hline alloy C & 0.055 & 0.237 & 0.008 & 0.009 & 0.023 & 0.024 & 0 & 0.002 & 0.011 & 0.013 & 0 \\
& $(0.26)$ & & & & & & & & & & \\
\hline
\end{tabular}

of ferrite from the one-dimensional interface velocity, a three-dimensional geometrical model is required. Such a model actually accomplishes the translation from the microscopic transformation behaviour to the macroscopic observation of the ferrite fraction.

In this paper the tetrakaidecahedron is used as a typical, regular Voronoi cell (see Fig. 2) to model the austenite grain geometry. As was shown in earlier work, due to its geometrical properties the tetrakaidecahedron is an ideal representation of an austenite grain for modelling the transformation behaviour ${ }^{6)}$ : the grain corners enable the modelling of heterogeneous nucleation as it is often reported in the literature, ${ }^{14,15}$ and by resizing the grain the influence of the austenite grain size on the transformation kinetics can be incorporated. It was shown in ref.() that the choice of model geometry for the austenite microstructure has significant effects on the calculated transformation kinetics.

In the model described here the ferrite phase is modelled to grow from the corners of the tetrakaidecahedron, at which nucleation is assumed to occur. The velocity $v$ is given by Eq. (2), being independent of the direction of the growth, and the ferrite will thus grow as segments of spheres. In every time step the ferrite fraction is determined by means of numerical integration.

\section{Experimental}

The transformation kinetics in three different lean C-Mn steel grades were investigated. The composition of the alloys is given in Table 1. The transformation kinetics were measured using a Bähr 805A dilatometer, on cylindrical specimens, $10 \mathrm{~mm}$ long with a diameter of $4 \mathrm{~mm}$. The specimens were austenitised at $1223 \mathrm{~K}$ and subsequently cooled at a cooling rate of either $0.05,0.3$, or $10 \mathrm{~K} / \mathrm{s}$. Alloy A has also been measured at a cooling rate of $60 \mathrm{~K} / \mathrm{s}$. Apart from the temperature-control thermocouple, a second thermo- 
couple, placed at $4 \mathrm{~mm}$ from the first, was used to check the temperature gradient along the length of the specimen. The measured temperature gradient was always smaller than 5 $\mathrm{K}$.

The volume fraction ferrite has been determined from the measured length change, taking into account the carbon enrichment of the austenite during the transformation and the difference between the specific volumes of ferrite and pearlite. ${ }^{16)}$

In order to determine the austenite grain size, samples for optical microscopy were prepared by thermal etching. The ferrite grain size was determined after etching with $2 \%$ nital. The determination of both the ferrite and austenite grain sizes was carried out by means of the mean linear intercept method.

\section{Results and Discussion}

Figure 3 shows two micrographs of the etched ferritic microstructure of alloy A after austenitisation and cooling

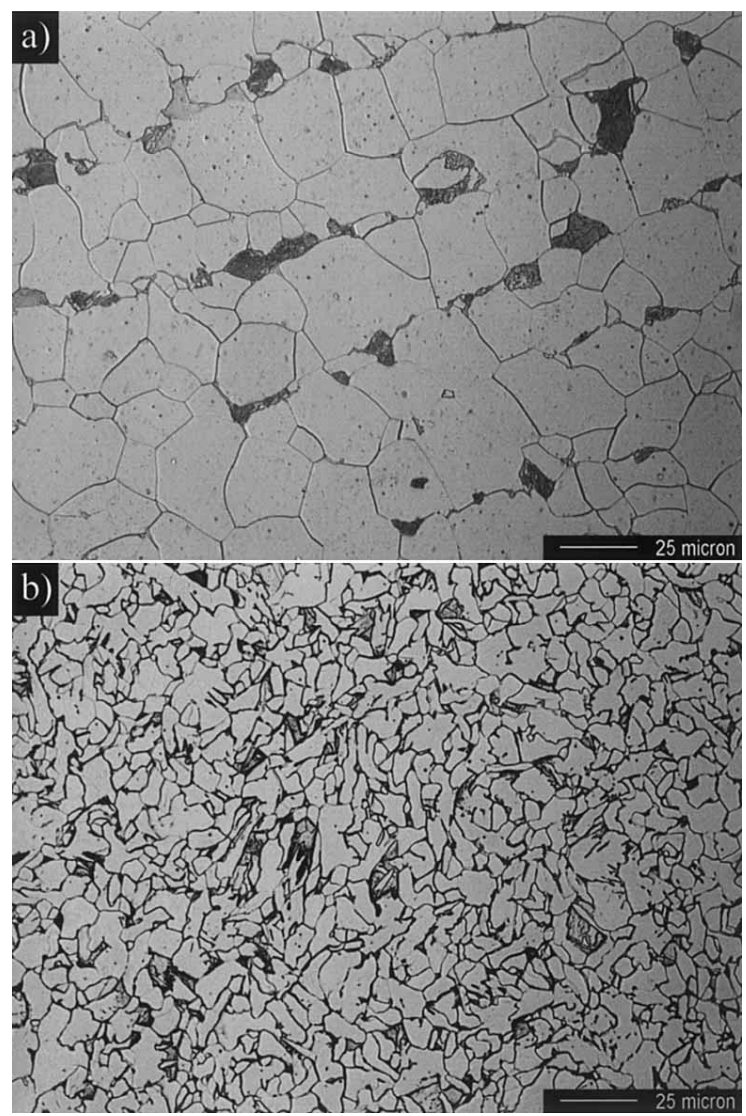

Fig. 3. Microstructure of alloy A, after cooling with a) $0.05 \mathrm{~K} / \mathrm{s}$, and b) $60 \mathrm{~K} / \mathrm{s}$ at 0.05 and $60 \mathrm{~K} / \mathrm{s}$, respectively. The microstructure resulting from the slow cooling shows distinct bands of pearlite. The microstructure attained with the high cooling rate has a more random distribution of pearlite in the ferrite matrix. This feature is also observed for the alloys B and C. Table 2 gives the results of the microstructural analysis for the three steel grades after cooling at the different rates, with $d^{i}$ the average grain size in phase $i$. The resulting nucleus density $n_{n}$ is calculated by $n_{n}=\left(1 / d^{\alpha}\right)^{3}$.

Figure 4 shows the measured ferrite volume fractions $f^{\alpha}$ for alloy $\mathrm{A}$ as a function of temperature. In this graph five curves are depicted. Four curves correspond to continuous cooling at $0.05,0.3,10$ and $60 \mathrm{~K} / \mathrm{s}$, the fifth curve is determined in a second experiment at a cooling rate of $0.3 \mathrm{~K} / \mathrm{s}$. During this experiment a $30 \mathrm{~min}$ isothermal hold at $1061 \mathrm{~K}$ was introduced. The curves belonging to the two lowest cooling rates coincide for $f^{\alpha}>0.5$. The transformation in the $0.3 \mathrm{~K} / \mathrm{s}$ experiment starts at a slightly lower temperature than in the $0.05 \mathrm{~K} / \mathrm{s}$ experiment. Due to the larger driving force the growth process is faster, and the $0.3 \mathrm{~K} / \mathrm{s}$ curve joins the $0.05 \mathrm{~K} / \mathrm{s}$ curve at $f^{\alpha} \approx 0.5$. From here on the transformation rate, as defined by volume fraction transformed per unit of temperature, is the same for the two cooling rates. The fact that the same development of the ferrite fraction occurs, can be explained by the transformation following the equilibrium state. In order to check this, the second experiment at $0.3 \mathrm{~K} / \mathrm{s}$ has been interrupted for $30 \mathrm{~min}$ at $1061 \mathrm{~K}$. The fractions ferrite as a function of temperature for the two $0.3 \mathrm{~K} / \mathrm{s}$ experiments coincide over the complete temperature range, as can be seen in Fig. 4. During

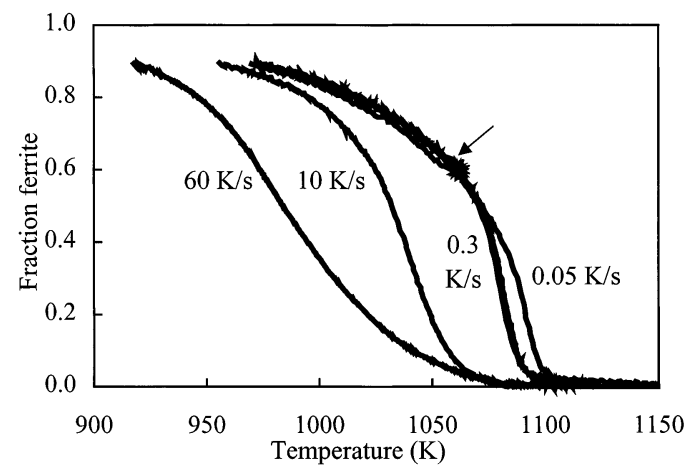

Fig. 4. Transformation curves, derived from dilatometry results, for steel grade A at controlled cooling rates of $0.05,0.3$, 10 and $60 \mathrm{~K} / \mathrm{s}$. An additional fraction curve corresponding to a cooling rate of $0.3 \mathrm{~K} / \mathrm{s}$, in which a $30 \mathrm{~min}$ isothermal hold at $1061 \mathrm{~K}$ was performed, is included. This curve coincides completely with the continuous cooled, $0.3 \mathrm{~K} / \mathrm{s}$, fraction curve. The observed ferrite fraction during the isothermal hold is indicated by the arrow.

Table 2. Microstructural data for the austenite and ferrite phase, as determined by optical microscopy, $d^{\gamma}$ is the average austenite grain diameter, $d^{\alpha}$ the average ferrite grain diameter and $n_{\mathrm{n}}$ the nucleus density.

\begin{tabular}{|c|c|c|c|c|c|c|c|c|c|}
\hline & & \multicolumn{2}{|c|}{$0.05 \mathrm{~K} / \mathrm{s}$} & \multicolumn{2}{|c|}{$0.3 \mathrm{~K} / \mathrm{s}$} & \multicolumn{2}{c|}{$10 \mathrm{~K} / \mathrm{s}$} & \multicolumn{2}{c|}{$60 \mathrm{~K} / \mathrm{s}$} \\
\hline & $d^{y}(\mu \mathrm{m})$ & $d^{\alpha}$ & $n_{\mathrm{n}}\left(10^{13} \mathrm{~m}^{-3}\right)$ & $d^{\alpha}$ & $n_{\mathrm{n}}\left(10^{13} \mathrm{~m}^{-3}\right)$ & $d^{\alpha}$ & $n_{\mathrm{n}}\left(10^{13} \mathrm{~m}^{-3}\right)$ & $d^{\alpha}$ & $n_{\mathrm{n}}\left(10^{13} \mathrm{~m}^{-3}\right)$ \\
$(\mu \mathrm{m})$ & & $(\mu \mathrm{m})$ & & $(\mu \mathrm{m})$ & & & \\
\hline $\mathrm{A}$ & 11 & 20 & 13 & 15 & 30 & 8 & 195 & 6 & 462 \\
\hline $\mathrm{B}$ & 11 & 25 & 6.4 & 25 & 6.4 & 8 & 195 & - & - \\
\hline $\mathrm{C}$ & 10 & 30 & 3.7 & 26 & 5.7 & 16 & 24 & - & - \\
\hline
\end{tabular}




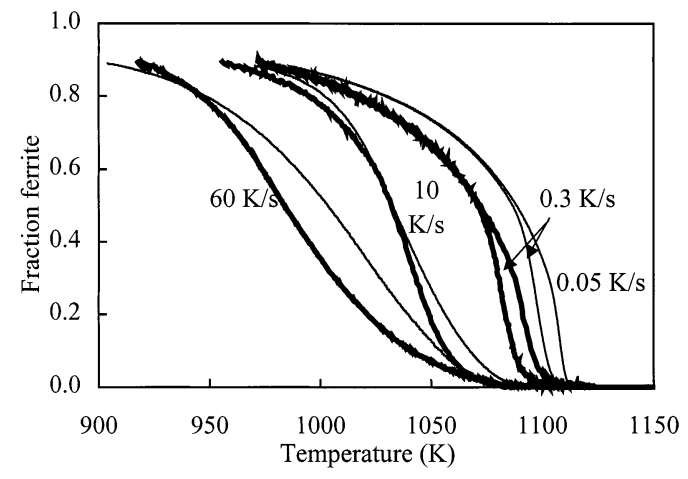

Fig. 5. Experimentally determined (thick lines) and simulated (thin lines) transformation curves for steel grade $\mathrm{A}$ at cooling rates of $0.05,0.3,10$ and $60 \mathrm{~K} / \mathrm{s}$.

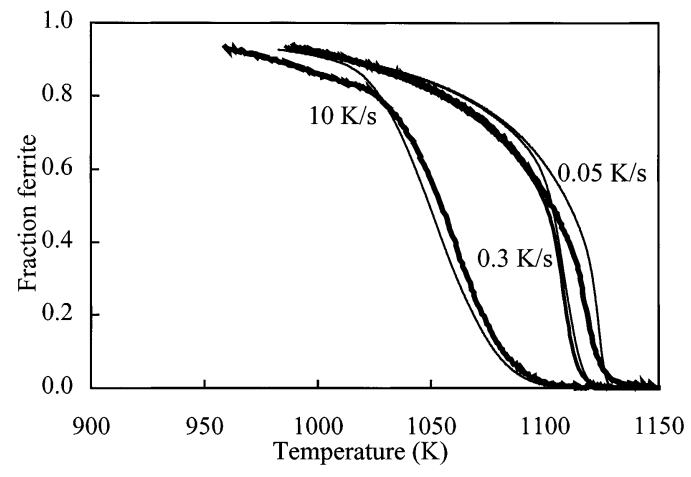

Fig. 6. Experimentally determined (thick lines) and simulated (thin lines) transformation curves for steel grade B at cooling rates of $0.05,0.3$ and $10 \mathrm{~K} / \mathrm{s}$.

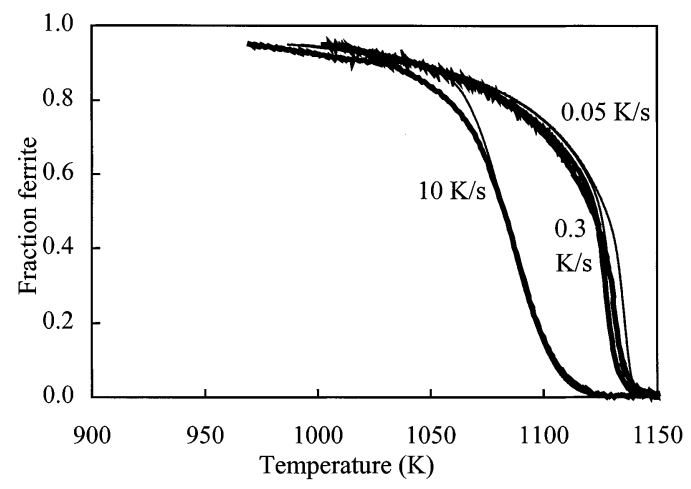

Fig. 7. Experimentally determined (thick lines) and simulated (thin lines) transformation curves for steel grade $\mathrm{C}$ at cooling rates of $0.05,0.3$ and $10 \mathrm{~K} / \mathrm{s}$.

the isothermal period, indicated by the arrow, there is no significant deviation of the fraction curve. The change in fraction which is observed during this period is caused by instrumental fluctuations. The transformation rate that appears in Fig. 4 gives the development of the ferrite fraction with temperature, or $d f^{\alpha} / d T$. This quantity gradually decreases with increasing cooling rate. The transformation rate with respect to time $\left(d f^{\alpha} / d t\right)$ increases with the cooling rate, caused by the increased undercooling that strongly enhances the driving force for transformation.

Figures 5, 6 and 7 give the experimentally determined ferrite fractions (thick lines) and the modelled curves (thin lines) for the alloys A, B and C, respectively. In the calculations, the nucleus density and the austenite grain size as
Table 3. The $M_{0}$-values (in $\mathrm{molm} / \mathrm{Js}$ ) used for the model curves in Figs. 5, 6 and 7.

\begin{tabular}{|l|l|l|l|l|}
\hline & $0.05 \mathrm{~K} / \mathrm{s}$ & $0.3 \mathrm{~K} / \mathrm{s}$ & $10 \mathrm{~K} / \mathrm{s}$ & $60 \mathrm{~K} / \mathrm{s}$ \\
\hline alloy A & 0.03 & 0.04 & 0.09 & 0.28 \\
\hline alloy B & 0.03 & 0.04 & 0.09 & \\
\hline alloy C & 0.03 & 0.06 & 0.18 & \\
\hline
\end{tabular}

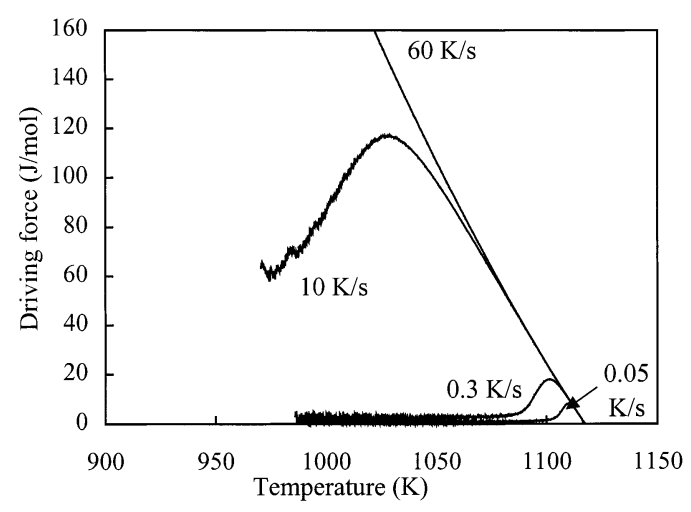

Fig. 8. The calculated driving forces corresponding to the model curves for alloy A in Fig. 5.

given in Table 2 are used, and the activation energy $Q$ is set to $140 \mathrm{~kJ} / \mathrm{mol}$. The mobility parameter $M_{0}$ is used as the single parameter to optimise the agreement between model and experimental curves. The resulting values are listed in Table 3.

The shape of the model transformation curves for all cooling rates corresponds well to the experimental fraction curves. In general, for all three alloys the correspondence between model and experiment is remarkable. All three alloys show the coincidence of the fraction curves for the two lowest cooling rates used, both in the experimental and in the model curves. It is seen, however, that the equilibrium fraction ferrite in the model curves is shifted towards higher values compared with the measured fraction. This difference is largest for alloy A (relatively high carbon and manganese concentrations) and smallest for alloy $\mathrm{C}$ (low carbon and manganese concentrations). For higher ferrite fractions the experimental and modelled fraction curves seem to approach each other. However on a temperature scale the difference between the modelled and the experimental fractions remains the same. The reason for this temperature shift between the experimental determined and calculated absolute ferrite fraction is unclear. Figure 8 shows the calculated driving force for the transformation in alloy A at the four different cooling rates. It is seen that the driving force strongly increases with increasing cooling rate, due to an increased lagging of the ferrite fraction with respect to the equilibrium fraction. At the two lowest cooling rates, after an early maximum in the driving force, the transformation proceeds according to equilibrium, and the driving force levels off to a small value that is related to the time step in the simulations.

A relative comparison between the three alloys shows the highest transformation start temperatures for steel grade $\mathrm{C}$. This occurs both for the experimental and the calculated fraction curves. Furthermore it is seen that for the higher cooling rates the correspondence between the experimental 
and calculated fraction curves for the carbon and manganese rich steel grade is less accurate. This is possible due to the fact that in the calculations nucleation is assumed to take place instantaneously at the $\mathrm{Ae}_{3}$-temperature. Since manganese is known to retard the nucleation kinetics, this assumption is expected to become less adequate for the steel grades in the order A, B, C.

Considering the $M_{0}$-values (Table 3) two trends can be observed: the value of $M_{0}$ increases with increasing cooling rate and with decreasing carbon content. These trends should be considered in view of the fact that $M_{0}$ is used as the only parameter to optimise the agreement between model and experimental curves; consequently, all factors influencing the overall transformation kinetics, but which have been disregarded in the model, will influence its value. The three main factors, viz. the diffusivity of carbon, the build-up of stresses at the $\alpha / \gamma$-interface, and the differences between the actual nucleus density and the density derived from the microstructures, will now be discussed.

For the model assumption that the carbon is homogeneously distributed in the austenite to be valid at each moment in time, it is required that the carbon diffusion is infinitely fast compared with the $\gamma / \alpha$-interface mobility. If the finite carbon diffusion plays a role in the determination of the $\gamma / \alpha$-interface velocity, it causes a carbon pile-up in the austenite in front of the transforming interface, leading to a lower driving force. This implies a slower transformation, and in the calculations a slower transformation is described by a lower $M_{0}$-value. A carbon pile-up at the interface is more likely to occur at higher cooling rates, since there will be less time for carbon to diffuse into the bulk, and with higher initial carbon concentrations, since the carbon concentration gradient will be less pronounced.

Ferrite has a larger specific volume than austenite, therefore during the transformation the material has to expand. This will occur via stresses surrounding the ferrite grains, which effectively corresponds to a lower driving force for the transformation, and thus causes a slower transformation. The effect of stress build-up at the interface is expected to increase with increasing cooling rate, since there is less time for recovery of the stress, and with increasing alloying content, since alloying elements slow down the recovery process.

Considering the nucleus density the following remarks can be made. The more nuclei are present, the faster the transformation will proceed. If, due to grain coarsening, the nucleus density derived by the present method (relating the $\alpha$ - and $\gamma$-microstructures) is too low, a too high velocity is attributed to the interface, since in the calculations the same overall transformation kinetics is to be achieved by a smaller number of ferrite grains. Grain coarsening is expected to be enhanced when more nuclei are formed, i.e. at higher cooling rates and lower alloying contents.

It is shown in the present study that the austenite to ferrite transformation is well described by the transformation model, in which the most important parameters, such as the austenite grain geometry, the nucleus density, and the driving force, based on chemical composition and temperature, are incorporated. An evaluation of the mobility of the interface with respect to cooling rate and carbon content in- dicates the grain coarsening to play a significant role in the transformation kinetics. This is derived from the increase of $M_{0}$ with increasing cooling rate and decreasing carbon concentration, a tendency that is expected from an increasing underestimation of the nucleus density, as discussed above. The effects of finite carbon diffusion and transformation stresses would lead to an opposite tendency.

\section{Conclusions}

An austenite-to-ferrite transformation model based on the concept of interface mobility has been tested for three commercial steel grades at cooling rates between 0.05 and $60 \mathrm{~K} / \mathrm{s}$. The model satisfactorily predicts the transformation rate at all cooling rates, including the ranges in which the transformation follows the equilibrium. For an optimal description of the fraction ferrite as a function of temperature for this series of experiments, a slight variation in $M_{0}$-values is used. The increasing tendency of $M_{0}$ with increasing cooling rate and decreasing carbon content appears to be caused by an underestimation of the nucleus density due to grain coarsening.

\section{Acknowledgments}

The IOP-Metals programme, Hoogovens Research and Development Center, and Erik Peekstok (Delft University of Technology) are acknowledged for their (financial) support, valuable discussions and contributions to the experimental work.

\section{REFERENCES}

1) J. W. Johnson and R. F. Mehl: Trans. AIME, 135 (1939), 416.

2) M. Avrami: J. Chem. Phys., 7 (1939), 1103; 8 (1940), 212; 9 (1941), 177.

3) C. Zener: J. Appl. Phys., 20 (1949), 950.

4) J. W. Christian: The Theory of Transformations in Metals and Alloys, 2nd ed. Pergamon Press, Oxford, (1981), 476.

5) G. P. Krielaart, J. Sietsma and S. van der Zwaag: Mat. Sci. Eng., A237 (1997), 216.

6) Y. van Leeuwen, S. I. Vooijs, J. Sietsma and S. van der Zwaag: Metall. Mater. Trans., 29A (1998), 2925.

7) S. I. Vooijs, Y. van Leeuwen, J. Sietsma and S. van der Zwaag: Metall. Mater. Trans. A, 31A (2000); J. J. Wits, T. A. Kop, Y. van Leeuwen, J. Sietsma and S. van der Zwaag: Mat. Sci. Eng. A, $\mathbf{A 2 8 3}$ (2000), 234.

8) M. Enomoto and H. I. Aaronson: Metall. Trans., 17A (1986), 1381, 1385, 1399.

9) R. Priestner and P. D. Hodgson: Mater. Sci. Technol., 8 (1992), 849.

10) R. Bengochea, B. Lopez and I. Gutierrez: Metall. Mater. Trans., 29A (1998), 417.

11) P. D. Hodgson, R. M. Trail, R. K. Gibbs, J. Grace and C. H. J. Davies: 2nd Int. Conf. on Modelling of Metal Rolling Processes, ed. by J. H. Beynon, P. Ingham, H. Teichert and K. Waterson, The Institute of Materials, London, (1996), 345.

12) M. Hillert: Metall. Trans., 6A (1975), 5.

13) G. P. Krielaart and S. van der Zwaag: Mater. Sci. Technol., 14 (1998), 10.

14) W. Huang and M. Hillert: Metall. Mater. Trans., 27A (1996), 480.

15) Ch. Zurek, E. Sachova and H. P. Hougardy: Abschlussbericht Project COSMOS (Max-Planck-Institut für Eisenforschung GmbH, Düsseldorf, Germany, (1993).

16) T. A. Kop, J. Sietsma and S. van der Zwaag: "Dilatometric Analysis of Phase Transformations in Hypo-eutectoid Steels", J. Mater. Sci., to be published. 